\title{
Many Particle Theory for the Luminescence, Characterization and Simulation of Quantum Well Laser Structures
}

\author{
M.F. Pereira Jr., \\ Instituto de Física, Universidade Federal da Bahia, 40210-340, Salvador BA Brazil \\ A. A. Bernussi, W. Carvalho Jr., Mario T. Furtado, and A. L. Gobbi \\ Laboratório de Optoelectrônica, LNLS, 13083-970, Campinas, SP, Brazil
}

Received on 23 April, 2001

\begin{abstract}
A photon Green's function theory is used to incorporate Bethe- Salpeter-like many body corrections in the the computations of nonlinear optical spectra of semiconductor quantum well laser materials. The numerical results presented reproduce several features found in the experimental characterization of the actual devices.
\end{abstract}

The simulation of semiconductor lasers is a fascinating challenge to modern Physics.[1] They operate in a highly excited regime, where many-particle effects play a dominant rule.[2] A previous approach for quantum well lasers has successfully described the combination of resonant cavity, band structure and many body effects in a temperature regime and for materials where a vertex-type of approximation still successfully describes the excited media.[3] There are however experimental conditions, specially at low temperatures, where higher order Coulomb corrections must be dealt with. A more general theory is thus required. [4, 5] In this paper we present solutions of a Bethe-Salpeter type of equation for quantum wells within a photon Green's functions approach capable of handling the resonant cavity microscopically, as well as quantum confinement and band structure effects. The microscopic approach consistently describes Pauli-blocking, the screening of the Coulomb interaction, Coulomb enhancement of the polarization function, and band gap shrinkage.

The many-body approach used here is based on Keldysh Green's functions for carriers $(G)$, photons $(D)$, and plasmons $(W)$ to describe the coupled lightexcited semiconductor system. [3, 4, 6, 7] Here, we just outline the method with words and a few representative equations. The Keldysh Green's function's time evolution is described by Dyson equations, characterized by free propagators $G_{o}^{-1}, D_{o}^{-1}, W_{o}^{-1}$, and selfenergies, $\Sigma, P$, and $p$, being the carrier self-energy, the transverse, and the longitudinal polarization functions, respectively. Detailed band-structure and quantumconfinement effects, obtained by solving the Luttinger
Hamiltonian, are included in the free-carrier propagator $G_{o}^{-1}$, and serve as the input for the solution of our many-body problem. Each of the self energies changes the bare into dressed propagators in a specific way.

The transverse polarization $P$ describes the optical response of the system, and can be written as a sum of an RPA term and a Coulomb-correlation contribution, expressed by the solutions of the (integro-differencial) Bethe-Salpeter equation,[4]

$P(1,1,2,2)=G(1,2) G(2,1)+G(1,3) G(4,1) P(3,4,2,2)$.

In order to describe light emission, we refer to the quantum mechanical Poynting vector. If we neglect resonant cavity effects, the PL spectrum is given essentially by the carrier recombination spectrum, described by $P^{<}(\omega)$. If we wish to describe the cavity, the photon Green's function presented here handles the problem in first principles. The polarization function satisfies the Kubo Martin Schwinger (KMS) sum rule. [2, 4] So, once $\operatorname{ImPr}$ is computed, the carrier recombination spectra is immediately obtained, ( $\mu$ is the total, electron + hole, chemical potential),

$$
P^{<}(\omega)=\frac{-2 i \operatorname{Im}\left\{P^{r}(\omega)\right\}}{1-\exp (\beta(\hbar \omega-\mu))}
$$

Fig. 1 compares the measured (symbols) photoluminescence (P.L.) with the numerical solutions of Eqs. $(1,2)$ with (solid) and without (dashed) inhomogeneous broadening. 


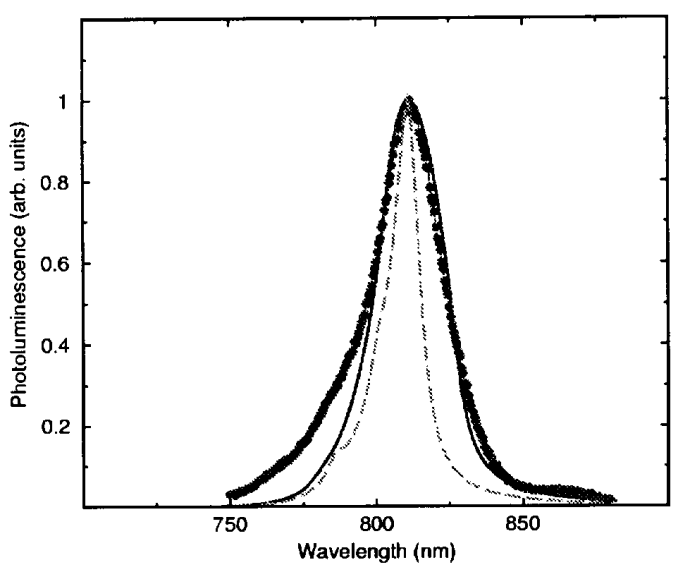

Figure 1. Photoluminescence spectra of a $30 \mathrm{~nm}$ InGaAsP quantum well at $300 \mathrm{~K}$. The symbols depict experimental results, while the solid and dashed curves are computed, respectively with and without inhomogeneous broadening.

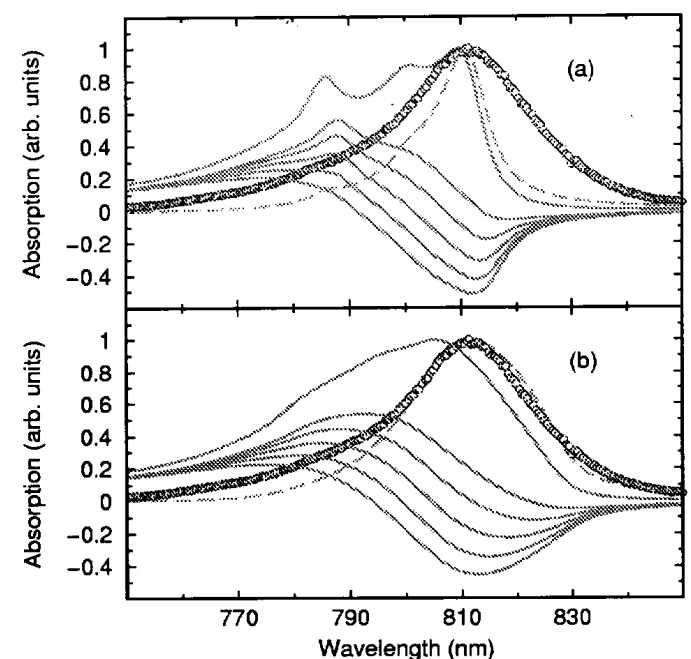

Figure 2. Evolution of the absorption spectra (solid) from the linear regime (top curve) to the gain region, as the excitation carrier density is increased the medium. The dashed curve is the P.L. computed for a low carrier density and the symbols are experimental data. All numerical parameters are equal to those of Fig.1 for a $30 \mathrm{~nm}$ InGaAsP quantum well at $300 \mathrm{~K}$. (a) and (b) are computed, respectively, without and with inhomogeneous broadening.

Further experimental investigations (not shown) suggest a sizable Stokes shift between P.L. and photocurrent measurements (P.C.). Although the resolution of the P.C. is not high enough at the moment for final conclusions, a Stokes shift can be described by the theory, as shown in Fig. 2. The inhomogeneous broadening is simulated here by means of a Gaussian distribution of the fundamental band gap, as depicted in Fig.2. for the computed nonlinear absorption spectra obtained from different carrier densities, from $\alpha(\omega) \sim c /(2 \omega \sqrt{\epsilon(\infty)}) \operatorname{ImP}^{r}(\omega)$, for the QW of Fig. 1. Note the increase of Stokes shift between absorption and emission in the inhomogeneously broadened curve (b) in comparison with (a). Possible explanations for the inhomogeneous broadening are: (i) Ordering in the quaternary alloy (InGaAsP), reducing the band gap, and increasing the Stokes shift between emission and absorption. (ii) Non- mixing of the quaternary alloy in the well and barrier regions, giving rise to gap energy modulation, broadening the emission peak. Both effects are strongly dependent on the growth temperature, and at that temperature, the well material would be out of the non- mixing region. However, both well and barrier could be ordered at that temperature. A more detailed experimental analysis is foreseen for a complete comparison with experiments.

The absorption spectra here contains only the first few transitions. A direct comparison with the full experimental photocurrent spectrum, requires all allowed transitions (not shown). The transitions depicted play the major role in the gain spectra, since the higher subbands are not appreciably populated.

Fig. 3 depicts the increasing Stokes shift with inhomogeneous broadening achieved here, by increasing the broadening of the Gaussian distribution.

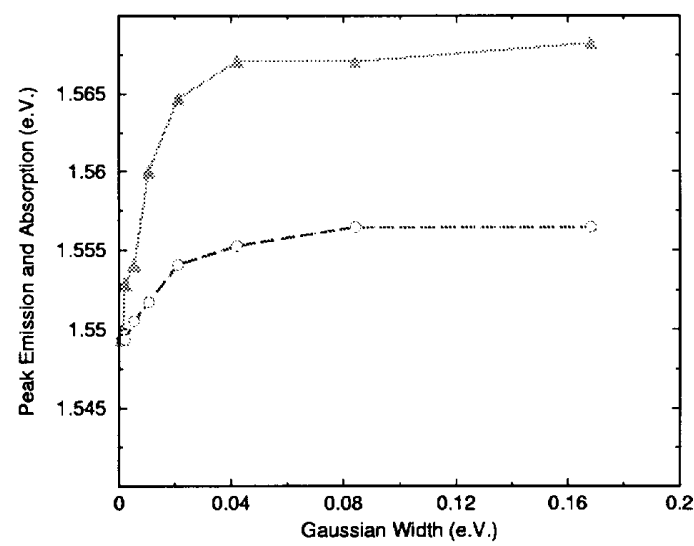

Figure 3. Linear Absorption peak (triangles) and Peak Luminescence (circles) as a function of inhomogeneous broadening, described by the width of the Gaussian distributions broadening.

In summary, regardless of the effective dimensionality of the system the microscopic mechanisms giving rise to optical gain, the semiconductor must be consistently treated as both resonator and gain medium. High density (e.g. Vertex) approximations, may be misleading, specially for systems with large exciton binding energy, and are avoided here, by means of a photon Green's function theory for quantum well laser spectra that allows the consistent inclusion of beyond RPA corrections in the polarization function by means of Bethe-Salpeter-Equation-like corrections. The numerical codes used here include a simplifying ansatz for the non- interacting polarization bubble used as input for the full numerical solution of the Bethe- Salpeter equation diagram that includes Coulomb- Many- Body corrections. The algorithm avoids a Kramers- Kronig integration in the loop and allows the reduction of a complex to a real matrix in the numerical matrix inversion computation that increases the speed of computation. Regardless of the simplification, the resulting equations can be used to predict a wealth of 
physical effects and make a starting point for simulating the operation of semiconductor lasers, amplifiers, and other hot-semiconductor-cavity optoelectronic devices, and the current numerical results successfully reproduce several operational characteristics of the lasers developed at Fundação CPQd-LNLS.

\section{Acknowledgments}

Research supported by Conselho Nacional de Pesquisas, CNPq of Brazil.

\section{References}

[1] P. Zory, Quantum Well Lasers, Academic Press, San Diego, 1993.
[2] R. Zimmermann, Many-Particle Theory of Highly Excited Semiconductors, "Teubner Texte zur Physik", Leipzig (1987).

[3] M.F. Pereira Jr. and K. Henneberger, Phys. Rev. B 53, 16485 (1996).

[4] M.F. Pereira Jr. and K. Henneberger, Phys. Rev. B 58, 2064 (1998)

[5] P. Michler, M.Vehse, J. Gutowski, M. Behringer, and D. Hommel, M. F. Pereira Jr., and K. Henneberger, Phys. Rev. B 58, 2055 (1998).

[6] K. Henneberger and S.W. Koch, Phys. Rev. Lett. 76, 1820 (1996).

[7] K. Henneberger and H. Haug, Phys. Rev. B 38, 9759 (1988). 